ORIGINAL ARTICLE

\title{
Apatinib monotherapy for advanced non-small cell lung cancer after the failure of chemotherapy or other targeted therapy
}

\author{
Zui Liu', Wei Ou', Ning Li ${ }^{2} \&$ Si-Yu Wang ${ }^{1}$ \\ 1 Department of Thoracic Surgery, Sun Yat-sen University Cancer Center, State Key Laboratory of Oncology in South China, Collaborative \\ Innovation Center for Cancer Medicine, Guangzhou, China \\ 2 Department of Breast Oncology, Sun Yat-Sen University Cancer Center, State Key Laboratory of Oncology in South China, Collaborative \\ Innovation Center for Cancer Medicine, Guangzhou, China
}

\author{
Keywords \\ Anti-VEGF; apatinib; efficacy; non-small cell \\ lung cancer.

\section{Correspondence} \\ Si-Yu Wang, Department of Thoracic Surgery, \\ Sun Yat-sen University Cancer Center, State \\ Key Laboratory of Oncology in South China, \\ Collaborative Innovation Center for Cancer \\ Medicine, 651 Dongfeng Road East, \\ Guangzhou 510060, China. \\ Tel: +862087343736 \\ Fax: +862087343628 \\ Email: wangsy@sysucc.org.cn
}

Received: 4 June 2018;

Accepted: 16 July 2018

doi: $10.1111 / 1759-7714.12836$

Thoracic Cancer 9 (2018) 1285-1290

\begin{abstract}
Background: Apatinib, a small-molecule inhibitor of vascular endothelial growth factor receptor 2 (VEGFR-2), has proven to be effective and safe for treating patients with advanced gastric cancer after second-line chemotherapy failure. As VEGFR-2 targeted therapy has made encouraging progress for the treatment of a broad range of malignancies, we explored the efficacy and safety of apatinib for the treatment of advanced non-small cell lung cancer after the failure of chemotherapy or other targeted therapy.

Methods: We retrospectively analyzed the data of 34 patients (11 with squamous carcinoma and 23 with adenocarcinoma) who were treated with apatinib alone in a daily oral dose of $250 \mathrm{mg}$ in the second-line or third-line setting from January 2016 to July 2017. The primary endpoint was progression-free survival (PFS).

Results: EGFR mutation or amplification was detected in 15 patients. The median PFS of the whole group was four months (95\% confidence interval 0.3-7.7). A partial response was observed in 2 patients (5.88\%) and stable disease in $19(55.88 \%)$. The disease control rate was $61.76 \%$. Common side effects of apatinib were hypertension $(n=12)$, hand-foot syndrome $(n=8)$, and proteinuria $(n=5)$, which accounted for $35.30 \%, 23.53 \%$, and $14.71 \%$, respectively, and no grade $3 / 4$ adverse reactions occurred. Apatinib toxicity was controllable and tolerable.

Conclusions: Apatinib appears to be effective and safe for advanced non-small cell lung cancer after the failure of chemotherapy or other targeted therapy.
\end{abstract}

\section{Introduction}

Lung cancer is the most common cancer with the highest mortality in men worldwide and has become the leading cause of cancer death among women in developed countries in recent years. ${ }^{1}$ In China, non-small cell lung cancer (NSCLC) accounts for more than $80 \%$ of lung cancer cases. ${ }^{2}$ The leading cause of cancer death among both men and women is cancer of the lung and bronchus, for which the 2015 age-standardized mortality rate in China was 733.3 per 100000 (509.3 per 100000 in men and 224.0 per 100000 in women). ${ }^{3}$ Patients with NSCLC are usually diagnosed at advanced stage. For advanced NSCLC patients with or without EGFR mutations, EGFR-tyrosine kinase inhibitors (TKIs) and chemotherapy are recommended as first-line treatment, respectively. ${ }^{4-10}$ After the failure of first-line therapy, second or third-line treatment does not currently yield acceptable progression-freesurvival (PFS) or overall survival (OS). ${ }^{11-13}$ Thirdgeneration EGFR-TKIs were not available in mainland China at the beginning of our research.

Vascular endothelial growth factor (VEGF) could stimulate angiogenesis, regulate vascular permeability, and 
inhibit apoptosis in endothelial cells. ${ }^{14}$ Bevacizumab, a humanized anti-VEGF monoclonal antibody, specifically binds to the VEGF-A protein, thereby inhibiting the process of angiogenesis. It has been recommended as first-line therapy for lung adenocarcinoma in the National Comprehensive Cancer Network (NCCN) Clinical Practice Guidelines in Oncology. Apatinib is a novel oral TKI that selectively inhibits VEGF receptor 2 (VEGFR-2), thus exerting an antiangiogenic effect and repressing tumor growth. ${ }^{14,15}$ Apatinib may be useful in circumventing multiple drug resistance (MDR) to other conventional chemotherapy drugs by inhibiting the transport function of ABCB1 and ABCG2-mediated MDR. ${ }^{16}$ Based on the results of two clinical trials, apatinib has been recommended to treat chemotherapy-refractory patients with metastatic gastric cancer or adenocarcinoma of the gastroesophageal junction. ${ }^{17,18}$

In this study, we evaluated the efficacy and toxicity of apatinib in advanced NSCLC patients after the failure of chemotherapy or EGFR-TKI therapy.

\section{Methods}

\section{Patient selection}

The data of 34 patients with stage IV or recurrent NSCLC treated from 28 January 2016 to 15 July 2017 were retrospectively analyzed. Patients were eligible if they had been histologically or cytologically diagnosed with lung squamous carcinoma or adenocarcinoma with confirmed EGFR mutations or $A L K$ rearrangement; and had an Eastern Cooperative Oncology Group (ECOG) performance status (PS) of 0-2. All histological diagnoses of NSCLC were made according to 2015 World Health Organization histopathological criteria. Tumors were classified according to the seventh edition American Joint Committee on Cancer Staging System.

The Ethics Committee of the Guangdong Association Study of Thoracic Oncology approved the study and all participants signed informed consent.

\section{Treatment}

Patients were treated with apatinib alone in daily oral dose of $250 \mathrm{mg}$ after the failure of previous therapy: patients with EGFR mutations received apatinib after EGFR-TKI therapy or EGFR-TKI therapy followed by chemotherapy; patients with wild-type EGFR received apatinib after first or second-line chemotherapy. The treatment cycle was 28 days, during which time patients took apatinib every day until disease progression or intolerable adverse events (AEs) occurred. No radiotherapy or other local therapy was offered during apatinib treatment.

\section{Response and toxicity evaluation}

Magnetic resonance imaging (MRI) or computed tomography (CT) scans of measurable lesions were assessed at the end of the first cycle, then every two cycles or earlier when significant signs of progression appeared. Response Evaluation Criteria in Solid Tumors (RECIST) was used to evaluate tumor responses to apatinib. Objective tumor responses included complete response (CR), partial response (PR), stable disease ( $\mathrm{SD})$, and progressive disease (PD). The disease control rate (DCR) was defined as the addition of objective response and stabilization rates $(\mathrm{CR}+\mathrm{PR}+\mathrm{SD})$. Blood pressure was checked weekly, blood counts and urinalysis were performed biweekly, and hepatic and renal function tests were performed monthly. Toxicities were evaluated according to National Cancer Institute Common Toxicity Criteria version 4.0 (CTC4.0).

\section{Statistical methods}

Progression-free survival was defined as the duration from the first date of apatinib administration to documented progression or mortality from any cause. Survival analysis was conducted using the Kaplan-Meier method and compared using a log-rank test. Statistical analysis was performed using SPSS version 23.0 (IBM Corp., Armonk, NY, USA). The median follow-up period was 11.4 months (range: 2.3-17.8). Follow-ups were conducted up to 15 July 2017.

\section{Results}

\section{Patient characteristics}

Thirteen patients were male and 21 were female. Ten had a smoking history and 24 had never smoked. Eleven patients had squamous carcinoma and 23 had adenocarcinoma. Twelve patients were detected as EGFR mutation positive (7 with deletions in exon 19, 4 with L858R in exon 21, 1 with L861Q in exon 21); three via fluorescence in situ hybridization as EGFR-positive. No patients had $A L K$ fusion gene rearrangements. Twenty-one patients were in stage IV and 13 were recurrent after radical surgery. Seventeen patients received apatinib as second-line therapy and 17 as third-line treatment. Nine patients had a PS of 0,22 had a PS of 1 , and 3 had a PS of 2 . The patient characteristics are listed in Table 1.

\section{Response to treatment}

The median PFS (mPFS) of the whole patient sample was four months (95\% confidence interval [CI] 0.3-7.7) (Fig 1). Among the 34 patients, PR was observed in 2 patients (5.88\%) and SD in 19 (55.88\%) (Fig 2). The DCR was $61.76 \%$. No significant correlation existed between PFS and gender $(P=0.45)$, age $(P=0.20)$, PS $(P=0.72)$, smoking 
Table 1 Baseline patient characteristics $(n=34)$

\begin{tabular}{ll}
\hline Characteristic & No. of patients (\%) \\
\hline Age & \\
Median (range), years & $55(30-74)$ \\
$>60$ & $11(32.35 \%)$ \\
$\leq 60$ & $23(67.65 \%)$ \\
Gender & \\
Female & $21(61.76 \%)$ \\
Male & $13(38.24 \%)$ \\
PS & \\
0 & $9(26.47 \%)$ \\
1 & $22(64.71 \%)$ \\
2 & $3(8.82 \%)$ \\
Smoking status & \\
Ever & $12(35.29 \%)$ \\
Never & $22(64.71 \%)$ \\
Pathology & \\
Adenocarcinoma & $23(67.65 \%)$ \\
Squamous cell carcinoma & $11(32.35 \%)$ \\
EGFR status & \\
Mutation & $12(35.29 \%)$ \\
Amplification & $3(8.82 \%)$ \\
Wild-type & $19(55.88 \%)$ \\
Line of apatinib therapy & \\
2 & $17(50 \%)$ \\
3 & $17(50 \%)$ \\
\hline PS, pefformance stas. &
\end{tabular}

PS, performance status.

status $(P=0.68)$, pathological subtype $(P=0.79), E G F R$ mutation status $(P=0.59)$, stage $(P=0.29)$, or line of therapy $(P=0.13)$. The results of univariate analysis are detailed in Table 2 .

Tumor response was evaluated according to the diameter of both measurable and unmeasurable lesions. Five

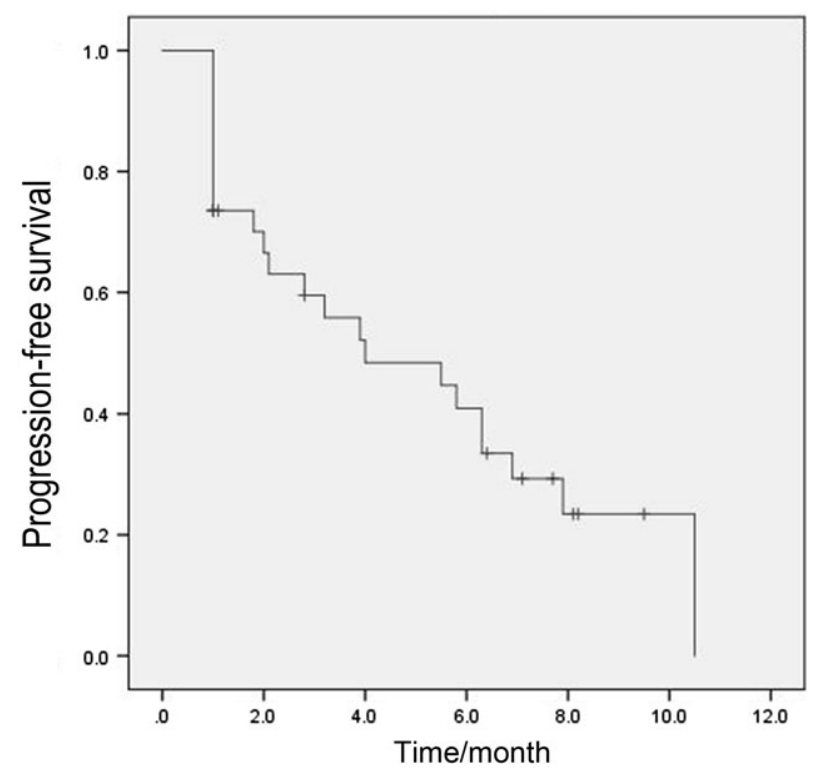

Figure 1 Kaplan-Meier estimates of progression-free survival. patients had unmeasurable lesions (pleural effusion with pleural nodules or with lung lesions that were difficult to measure).

\section{Safety and toxicity}

The common side effects of apatinib were hypertension $(n=12)$, hand-foot syndrome $(n=8)$, proteinuria $(n=5)$, and fatigue $(n=4)$, which accounted for $35.30 \%, 23.53 \%$, $14.71 \%$, and $11.76 \%$. No grade $3 / 4$ adverse reactions occurred. Six of the patients did not experience any AEs during the research period. No hematologic, liver, or renal toxicities were observed. No patients withdrew as a result of AEs. The adverse events are listed in Table 3.

\section{Discussion}

Our results show that $250 \mathrm{mg}$ daily apatinib for advanced NSCLC is effective and safe. EGFR-TKI therapy is recommended as first-line treatment or as second-line setting after chemotherapy for advanced NSCLC patients with EGFR mutations. Platinum-based doublet chemotherapy is recommended as first-line treatment and single-agent chemotherapy as second-line treatment for patients with wildtype EGFR and $A L K$-negative tumors. Zhang et al. conducted a phase II trial to determine whether apatinib could improve PFS compared to a placebo in patients with advanced non-squamous NSCLC who had failed two lines of therapy. ${ }^{19}$ Ninety patients were randomized to apatinib $750 \mathrm{mg}$ until disease progression or unacceptable toxicity. The trial showed that apatinib has a substantial clinical effect without severe additional AEs in patients with advanced NSCLC. Some of the patients in our sample declined chemotherapy as second-line treatment after EGFR-TKIs or chemotherapy. Third generation EGFRTKIs were not available in mainland China at the beginning of our follow-up; therefore, we administered apatinib as second-line treatment for these patients.

Progression-free survival in our patient sample was four months and the DCR was $61.76 \%$. Maruyama et al. reported better mPFS ( 2 months) than PFS (1.4 months) of second and third-line docetaxel in a phase III clinical trial. $^{13}$ The mPFS in our study was 6.3 months in the second-line setting, which was superior to those reported in two phase III clinical trials. ${ }^{11,12}$ In the CTONG0806 study, pemetrexed was administered as second-line treatment in advanced non-squamous NSCLC patients with wild-type EGFR and the mPFS was 4.8 months. ${ }^{11}$ The ETOP study showed an mPFS of 4.1 months in advanced squamous cell NSCLC patients treated with docetaxel as second-line setting. ${ }^{12}$ In our patient sample, the mPFS in squamous cell NSCLC patients was 5.5 months (95\% CI $0-12.7)$ and in adenocarcinoma NSCLC patients 6.3 months (95\% CI $0-12.8$ ) as a second-line setting. These 


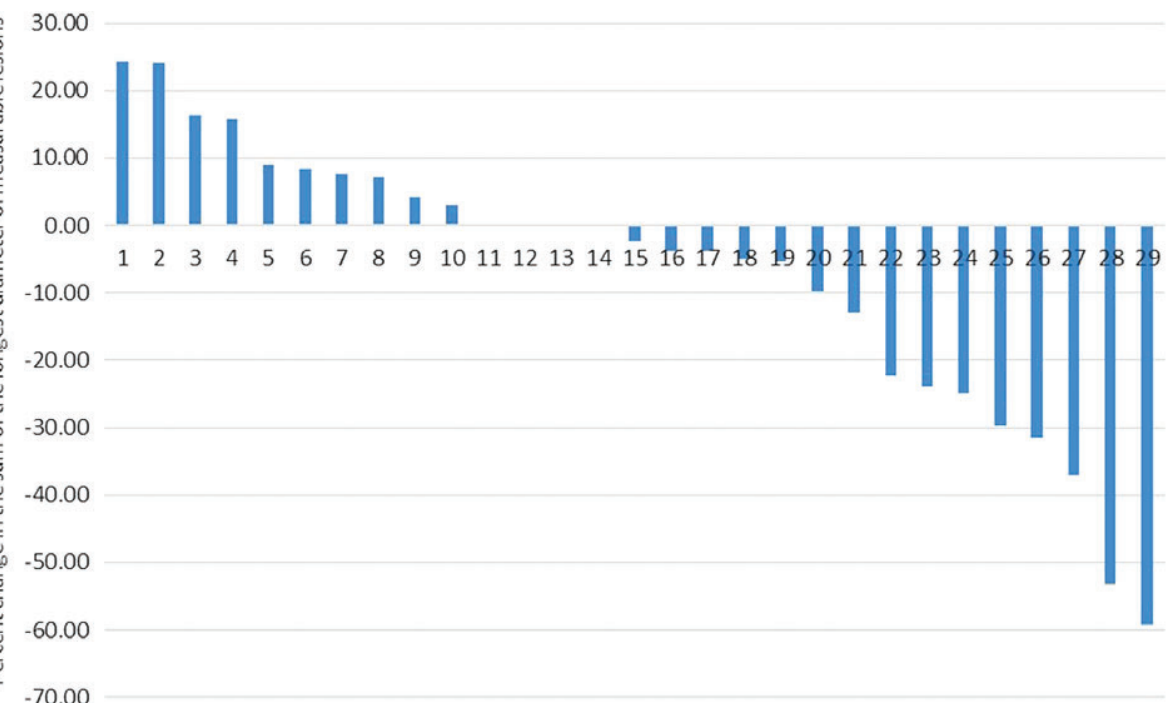

Figure 2 Waterfall plot of measurable lesion response.

Table 2 Univariate analysis of PFS

\begin{tabular}{|c|c|c|c|}
\hline Characteristic & mPFS & $95 \% \mathrm{Cl}$ & $P$ \\
\hline Gender & & & 0.446 \\
\hline Male & 3.2 & $0.1-6.3$ & \\
\hline Female & 5.8 & $3.4-8.1$ & \\
\hline Age, years & & & 0.203 \\
\hline$>60$ & 3.2 & $0.0-6.7$ & \\
\hline$\leq 60$ & 4 & $0.0-9.8$ & \\
\hline PS & & & 0.726 \\
\hline 0 & 6.3 & $3.1-9.5$ & \\
\hline 1 & 2.8 & $0.0-6.1$ & \\
\hline 2 & 7.9 & $0.4-15.4$ & \\
\hline Line of therapy & & & 0.134 \\
\hline 2 & 6.3 & $2-10.6$ & \\
\hline 3 & 3.9 & $0.9-6.9$ & \\
\hline Smoking status & & & 0.682 \\
\hline Ever & 3.2 & $2.1-4.3$ & \\
\hline Never & 5.8 & $2.0-9.6$ & \\
\hline Histology & & & 0.789 \\
\hline Adenocarcinoma & 4 & $0.5-7.5$ & \\
\hline Squamous cell & 2.1 & $0-6.8$ & \\
\hline EGFR status & & & 0.843 \\
\hline Mutation & 4 & $1.2-6.7$ & \\
\hline Amplification & 6.3 & $\dagger$ & \\
\hline Wild-type & 5.5 & $0.8-10.2$ & \\
\hline
\end{tabular}

†Only three patients harbored EGFR amplification and one did not arrive at the endpoint. $\mathrm{Cl}$, confidence interval; mPFS, median progression-free-survival; PS, performance status.

Table 3 Apatinib-related adverse events

\begin{tabular}{lccc}
\hline & \multicolumn{2}{c}{ No. of adverse events } & \\
\cline { 2 - 3 } Type of adverse event & Grade 1 & Grade 2 & Percentage (\%) \\
\hline Hypertension & 9 & 3 & 35.30 \\
Hand-foot syndrome & 5 & 3 & 23.53 \\
Proteinuria & 3 & 2 & 14.71 \\
Fatigue & 1 & 3 & 11.76 \\
\hline
\end{tabular}

results show that apatinib might have similar or even better efficacy than single-agent chemotherapy as second-line treatment in advanced NSCLC. In recent research, apatinib was administered at a dose of $500 \mathrm{mg}$ in third-line or further settings for advanced NSCLC. ${ }^{20}$ The median PFS was 4.2 months and the DCR was $61.9 \%$, which was consistent with our results.

Li et al. used apatinib in a third-line setting for patients with metastatic gastric cancer at a dose of $850 \mathrm{mg}$ once daily or $425 \mathrm{mg}$ twice daily. ${ }^{17}$ The incidence of hypertension, proteinuria, and hand-foot syndrome was $40.43 \%$, $27.66 \%$, and $25.53 \%$ in the $850 \mathrm{mg}$ once daily and $39.13 \%$, $34.78 \%$, and $45.65 \%$ in the $425 \mathrm{mg}$ twice-daily groups, respectively. Grade 3 to 4 hand-foot syndrome and hypertension occurred in more than $10 \%$ of patients. $\mathrm{Hu}$ et al. recommend a dose of apatinib $500 \mathrm{mg}$ /day rather than $750 \mathrm{mg} /$ day for pretreated patients with metastatic triplenegative breast cancer, because of the toxicity associated with the higher dose. ${ }^{21}$ At a dosage of $500 \mathrm{mg} /$ day, the incidence of hypertension, proteinuria, and hand-foot syndrome was $64.4 \%, 52.6 \%$, and $49.2 \%$, respectively. The AEs observed in our patient sample included hypertension, proteinuria, and hand-foot syndrome. We observed more mild to moderate AEs at a dose of $250 \mathrm{mg}$ compared to the $500 \mathrm{mg}$ used by Song et al. ${ }^{20}$ Zeng et al. reported results of four advanced lung adenocarcinoma patients with KRAS mutations treated with apatinib at a dose of $250 \mathrm{mg} / \mathrm{d} .^{22}$ Only one patient showed grade 1 hoarseness and hemoptysis. Our results indicate that $250 \mathrm{mg} / \mathrm{d}$ might be an optional dosage in advanced NSCLC patients. However, further large-scale clinical trials are needed to verify the most suitable dosage of apatinib.

The major limitations of the present study are its small sample size and its retrospective nature. In addition, we observed cavities in the tumor after the administration of 
apatinib in three patients. As there are currently no evaluation criteria for tumor cavities, we evaluated the tumor diameter ignoring the cavity. During anti-angiogenesis therapy, patients with advanced lung cancer commonly developed cavitation in their lung lesions. ${ }^{23} \mathrm{~A}$ cavernous tumor response could be assessed by volume assessment for target lesions or fluorodeoxyglucose metabolism. ${ }^{24,25}$ We expect that evaluation criteria for tumor cavities is forthcoming. Although the dose of $250 \mathrm{mg}$ apatinib in a second-line setting adopted in this study is not widely recommended, the safety and efficacy observed were promising.

Based on our results, it will be interesting to view further research of apatinib combined with chemotherapy in a first-line setting for advanced NSCLC.

\section{Acknowledgment}

We would like to thank the participating patients for their contribution to this study.

\section{Disclosure}

No authors report any conflict of interest.

\section{References}

1 Torre LA, Bray F, Siegel RL, Ferlay J, Lortet-Tieulent J, Jemal A. Global cancer statistics, 2012. CA Cancer J Clin 2015; 65: 87-108.

2 Zhi XY, Zou XN, Hu M, Jiang Y, Jia MM, Yang GH. Increased lung cancer mortality rates in the Chinese population from 1973-1975 to 2004-2005: An adverse health effect from exposure to smoking. Cancer 2015; 121 ((Suppl 17)): 3107-12.

3 Chen WQ, Zheng RS, Baade PD et al. Cancer statistics in China, 2015. CA Cancer J Clin 2016;66:115-32.

4 Maemondo M, Inoue A, Kobayashi K et al. Gefitinib or chemotherapy for non-small-cell lung cancer with mutated EGFR. N Engl J Med 2010; 362: 2380-8.

5 Mitsudomi T, Morita S, Yatabe Y et al. Gefitinib versus cisplatin plus docetaxel in patients with non-small-cell lung cancer harbouring mutations of the epidermal growth factor receptor (WJTOG3405): An open label, randomised phase 3 trial. Lancet Oncol 2010; 11: 121-8.

6 Mok TS, Wu YL, Thongprasert S et al. Gefitinib or carboplatin-paclitaxel in pulmonary adenocarcinoma. $N$ Engl J Med 2009; 361: 947-57.

7 Rosell R, Carcereny E, Gervais R et al. Erlotinib versus standard chemotherapy as first-line treatment for European patients with advanced EGFR mutation-positive non-smallcell lung cancer (EURTAC): A multicentre, open-label, randomised phase 3 trial. Lancet Oncol 2012; 13: 239-46.
8 Sequist LV, Yang JC, Yamamoto N et al. Phase III study of afatinib or cisplatin plus pemetrexed in patients with metastatic lung adenocarcinoma with EGFR mutations. $J$ Clin Oncol 2013; 31: 3327-34.

$9 \mathrm{Wu}$ YL, Zhou C, Hu CP et al. Afatinib versus cisplatin plus gemcitabine for first-line treatment of Asian patients with advanced non-small-cell lung cancer harbouring EGFR mutations (LUX-Lung 6): An open-label, randomised phase 3 trial. Lancet Oncol 2014; 15: 213-22.

10 Zhou C, Wu YL, Chen G et al. Erlotinib versus chemotherapy as first-line treatment for patients with advanced EGFR mutation-positive non-small-cell lung cancer (OPTIMAL, CTONG-0802): A multicentre, open-label, randomised, phase 3 study. Lancet Oncol 2011; 12: 735-42.

11 Zhou Q, Cheng Y, Yang JJ et al. Pemetrexed versus gefitinib as a second-line treatment in advanced nonsquamous nonsmall-cell lung cancer patients harboring wild-type EGFR (CTONG0806): A multicenter randomized trial. Ann Oncol 2014; 25: 2385-91.

12 Peters S, Stahel RA, Dafni U et al. Randomized phase III trial of erlotinib versus docetaxel in patients with advanced squamous cell non-small cell lung cancer failing first-line platinum-based doublet chemotherapy stratified by VeriStrat good versus VeriStrat poor. The European Thoracic Oncology Platform (ETOP) EMPHASIS-lung trial. J Thorac Oncol 2017; 12: 752-62.

13 Maruyama R, Nishiwaki Y, Tamura T et al. Phase III study, $\mathrm{V}-15-32$, of gefitinib versus docetaxel in previously treated Japanese patients with non-small-cell lung cancer. J Clin Oncol 2008; 26: 4244-52.

14 Tian S, Quan H, Xie C et al. YN968D1 is a novel and selective inhibitor of vascular endothelial growth factor receptor-2 tyrosine kinase with potent activity in vitro and in vivo. Cancer Sci 2011;102:1374-80.

15 Ding J, Chen X, Gao Z et al. Metabolism and pharmacokinetics of novel selective vascular endothelial growth factor receptor-2 inhibitor apatinib in humans. Drug Metab Dispos 2013;41:1195-210.

16 Mi YJ, Liang YJ, Huang HB et al. Apatinib (YN968D1) reverses multidrug resistance by inhibiting the efflux function of multiple ATP-binding cassette transporters. Cancer Res 2010; 70: 7981-91.

17 Li J, Qin S, Xu J et al. Apatinib for chemotherapy-refractory advanced metastatic gastric cancer: Results from a randomized, placebo-controlled, parallel-arm, phase II trial. J Clin Oncol 2013; 31: 3219-25.

$18 \mathrm{Li}$ J, Qin S, Xu J et al. Randomized, double-blind, placebocontrolled phase III trial of apatinib in patients with chemotherapy-refractory advanced or metastatic adenocarcinoma of the stomach or gastroesophageal junction. J Clin Oncol 2016; 34: 1448-54.

19 Zhang L, Shi MQ, Huang C et al. A phase II, multicenter, placebo-controlled trial of apatinib in patients with advanced nonsquamous non-small cell lung cancer (NSCLC) after two previous treatment regimens. J Clin Oncol 2012; 30 (15 Suppl): Abstract 7548. 
20 Song Z, Yu X, Lou G, Shi X, Zhang Y. Salvage treatment with apatinib for advanced non-small-cell lung cancer. Onco Targets Ther 2017; 10: 1821-5.

$21 \mathrm{Hu}$ X, Zhang J, Xu B et al. Multicenter phase II study of apatinib, a novel VEGFR inhibitor in heavily pretreated patients with metastatic triple-negative breast cancer. Int $J$ Cancer 2014; 135: 1961-9.

22 Zeng DX, Wang CG, Huang JA, Jiang JH. Apatinib in the treatment of advanced lung adenocarcinoma with KRAS mutation. Onco Targets Ther 2017; 10: 4269-72.

23 Nishino M, Cryer SK, Okajima Y et al. Tumoral cavitation in patients with non-small-cell lung cancer treated with antiangiogenic therapy using bevacizumab. Cancer Imaging 2012; 12: 225-35.

24 Crabb SJ, Patsios D, Sauerbrei E et al. Tumor cavitation: Impact on objective response evaluation in trials of angiogenesis inhibitors in non-small-cell lung cancer. J Clin Oncol 2009; 27: 404-10.

25 Nguyen NC, Abhishek K, Nyon S, Farghaly HR, Osman MM, Reimers HJ. Are there radiographic, metabolic, and prognostic differences between cavitary and noncavitary nonsmall cell lung carcinoma? A retrospective fluorodeoxyglucose positron emission tomography/computed tomography study. Ann Thorac Med 2016; 11: 49-54. 